\title{
The utility of a blood culture database to identify patients suitable for outpatient parenteral antibiotic treatment
}

\author{
Mark Melzer, ${ }^{1}$ Liana Macpherson, ${ }^{2}$ Catherine Welch ${ }^{3}$
}

${ }^{1}$ Royal London Hospital, Barts Health NHS Trust, London, UK ${ }^{2}$ Royal London Hospital, London, UK

${ }^{3}$ Department of Epidemiology and Public Health, University College London, London UK

Correspondence to Dr Mark Melzer, Consultant in Infection, Royal London Hospital, Barts Health NHS Trust, 3'rd floor Pathology and Pharmacy Building, 80 Newark St., London E1 2ES, UK; mark.melzer1@bartshealth. nhs.uk

Received 12 August 2016 Revised 29 September 2016 Accepted 5 October 2016 Published Online First

7 November 2016
ABSTRACT

Background The clinical and cost-effectiveness of outpatient parenteral antimicrobial therapy (OPAT) services are well described. We used a blood culture database as a novel approach to case finding and determined its utility in identifying inpatients suitable for OPAT.

Methods From December 2012 to November 2013, consecutive adult inpatients with bacteraemia, and those recruited to OPAT, were prospectively studied. Univariate and multivariate logistic regression analysis were used to investigate the association between bacteraemic patient characteristics and OPAT recruitment.

Results There were 470 bacteraemic and 134 OPAT patients. The blood culture database identified 22 (16.4\%; Cl 10.5 to 23.6) additional patients suitable for OPAT, $4.7 \%(95 \% \mathrm{Cl} 3.0 \%$ to $7.0 \%)$ of the total bacteraemic cohort. $20(90.9 \%)$ of these patients had community-acquired bacteraemia. Bacteraemic patients with urinary tract infections (UTIs), 11/157 (7.0\%; 95\% Cl $3.5 \%$ to $12.2 \%$ ) were most commonly recruited to OPAT and Escherichia coli was the most common blood culture isolate. In the E. coli bacteraemic subgroup, extended-spectrum $\beta$-lactamase (ESBL) producers were significantly higher in the OPAT group, compared with the non-OPAT group, $9 / 11(81.8 \%)$ vs $17 / 192(8.9 \%)$, $\mathrm{p}<0.001$. Among OPAT patients, there were no deaths within 30 days and no significant difference in relapse rates between bacteraemic and non-bacteraemic patients, $1 / 22$ (4.6\%) vs 5/112 (4.5\%). In logistic regression analysis, there were no patient characteristics in the bacteraemic cohort that predicted recruitment to OPAT. In a subgroup analysis of patients with Gramnegative bacteraemia, ESBL production was strongly associated with OPAT recruitment, OR $5.85(95 \% \mathrm{Cl}$ 1.94 to 17.58), $p=0.002$.

Conclusions A blood culture database proved a useful adjuvant to a clinical referral system, particularly for patients with community onset, multidrug resistant UTIs caused by ESBL producing E. coli. All bacteraemic patients recruited to OPAT received treatment safely and had good clinical outcomes.

\section{INTRODUCTION}

Outpatient parenteral antibiotic treatment (OPAT) enables patients to receive treatment with intravenous antibiotics in their home or in an ambulatory care setting rather than in hospital. It is widely used in the UK and is associated with admission avoidance, decreased length of inpatient stay, cost savings and high levels of patient satisfaction. ${ }^{1-5}$ In carefully selected patients, outcomes are comparable with hospital based treatment ${ }^{6} 7$ and national guidelines assist with service delivery, development and good clinical practice. OPAT is also part of the UK government's healthcare strategy of moving hospital services into the community so OPAT is likely to continue to expand over the next 5 years. ${ }^{8}$

While there is much published literature on the success of OPAT services, there is little on methods used to identify patients. Traditionally, patient identification can be passive, waiting for a referral, or active, targeting specific specialties (eg, orthopaedics or acute assessment units). Often referrals are unsuitable for several reasons. These include patients living outside designated postcodes, inability to attend hospital for treatment on a daily basis, being elderly with multiple comorbidities that necessitate prolonged hospital stay, difficulty with vascular access and having conditions treatable with oral antibiotics. Bacteraemic patients feature in some OPAT populations although it is unclear the site of infection causing bacteraemias and how these patients were recruited. ${ }^{7-11}$ Overall, there is little in the literature on the value of microbiological results in patient identification with the emphasis on clinical assessment and the safe delivery of treatment. ${ }^{7}{ }^{12}$ We used an existing blood culture database as a novel approach for recruitment, and determined its utility in identifying additional adult inpatients suitable for OPAT.

\section{PATIENTS AND METHODS}

\section{Study setting}

This study was undertaken at the Royal London Hospital (RLH), Barts Health NHS Trust. The RLH serves a diverse population of about 250000 patients in Tower Hamlets, East London and is a regional referral centre for the North-East London sector. In addition to accident and emergency, general medicine, surgery, paediatric and maternity services, the RLH has 60 high-dependency and critical care beds (including neurosurgical, renal, and obstetric and gynaecological beds), specialist wards for renal transplant and haemodialysis patients, and a high-level intensive care unit.

\section{Study population}

From December 2012 to November 2013, consecutive bacteraemic inpatients and patients recruited to OPAT were prospectively studied. Patients aged $<16$ years were excluded.

\section{Bacteraemia cohort and definitions}

In bacteraemic patients, age, gender, inpatient specialty, site of infection, organism, susceptibility profile and mortality related outcomes were 
recorded. Bacteraemia was considered significant if a blood culture was isolated from a patient with a compatible clinical syndrome that was unlikely to be a skin or environmental contaminant. This was based upon the patient's history, examination, response to antimicrobial therapy and bacterial isolates from other body sites. ${ }^{13}$ Specialties at the time of bacteraemia were categorised as medicine, surgery (including orthopaedics), critical care, and obstetrics and gynaecology. For hospital-acquired or device related bacteraemia, the Centres for Disease Control and Prevention definitions were used to define the sites of infection ${ }^{14}$ and for community-onset bacteraemia, sites were defined following clinical, microbiological and radiological assessment. Bacteraemia in patients with an unknown source were classified as undefined.

\section{Microbiology data}

Blood cultures were analysed using an automated system BacT/ ALERT3D (bioMerieux, Mary l'Etoile, France). Isolates were identified using either the VITEK MS system (bioMerieux, Mary l'Etoile, France, database V.2.0) or Bruker Biotyper (Bruker Daltonic, Leipzig, Germany, software V.3.0) MALDI-TOF MS systems according to the manufacturer's instructions and the laboratory standard operating procedures. Susceptibility testing was performed on the Microscan walkAway system (Siemens Healthcare Diagnostics, Deerfield, Illinois, USA).

\section{OPAT cohort, data collection and ascertainment}

Over the same period age, gender, site of infection, medical specialty, presence of bacteraemia, duration of treatment and outcomes were recorded for all patients recruited to OPAT. Where possible, patients were treated with intravenous antibiotics once daily. Patients under 16 years of age were excluded. Patients were recruited by referrals from inpatient teams or general practitioners. or actively sought by attendance at acute assessment unit board rounds or attendance at multidisciplinary team meetings (eg, orthopaedics). In addition, some OPAT referrals were made as a consequence of blood culture results. These results prompted assessment for OPAT suitability by either an infection specialist (including a microbiologist) or the patient's clinical team. Patients received treatment in their homes or via a fast response nursing team in an ambulatory care setting.

\section{Statistical analysis}

We analysed the characteristics of bacteraemic patients (age, gender, place of acquisition, inpatient specialty, site of infection and mortality), comparing those who received and did not receive OPAT. For patients recruited to OPAT, we compared patient characteristics and duration of intravenous treatment for bacteraemic and non-bacteraemic patients. We also describe bacteraemic isolates from patients who received OPAT and compared these to patients who did not receive OPAT.

Quantitative data are presented as numbers and percentages. Associations between two categorical variables were tested using the Pearson's $\chi^{2}$ test and continuous variables using t-tests. As patients may present with more than one bacteraemic episode, we used number of patients as the denominator to calculate percentages for patient characteristics and number of bacteraemic episodes as the denominator for infection characteristics.

Univariate and multivariate logistic regression analyses were used to examine the association between age, gender, inpatient specialty and site of infection, and recruitment to OPAT in bacteraemic patients. As all OPAT bacteraemic episodes were community-onset, we could not adjust for place of acquisition because there were no OPAT patients with hospital-acquired bacteraemia. In a subgroup analysis of patients with Gram-negative bacteraemia, we also examined the association between Escherichia coli infection and extended-spectrum $\beta$-lactamase (ESBL) production, and recruitment to OPAT. Generalised estimating equations were used to account for dependency between multiple bacteraemic episodes for patients in the univariate and multivariate analyses. Data were analysed using Stata SE (V.13.1).

\section{Clinical governance}

The clinical governance audit committee of Barts Health NHS Trust approved the study.

\section{RESULTS}

Over the 12-month period, the number of inpatients with bacteraemia and patients recruited to OPAT are illustrated in figure 1 .

\section{Bacteraemic patients}

There were 470 patients with bacteraemia yielding 556 positive blood cultures. Patient demographic, clinical and mortality data are summarised in table 1 . Twenty-two $(4.7 \%$; 95\% CI 3.0\% to $7.0 \%$ ) bacteraemic patients were recruited to OPAT. All patients recruited had community-onset infection (either communityacquired or healthcare-associated). Of these, 20 had communityacquired bacteraemia, equivalent to $20 / 200(10.0 \%$; $95 \% \mathrm{CI}$ $6.2 \%$ to $15.0 \%)$ of all community-acquired bacteraemic episodes.

Compared with surgical patients, significantly more medical patients were recruited to OPAT. Outpatient treatment episodes were most common for urinary tract infection (UTI), 11/157 $(7.0 \% ; 95 \%$ CI $3.5 \%$ to $12.2 \%)$, skin and soft tissue infection

Figure 1 Inpatients with bacteraemia and patients recruited to outpatient parenteral antibiotic treatment (OPAT) between December 2012 and 2013.

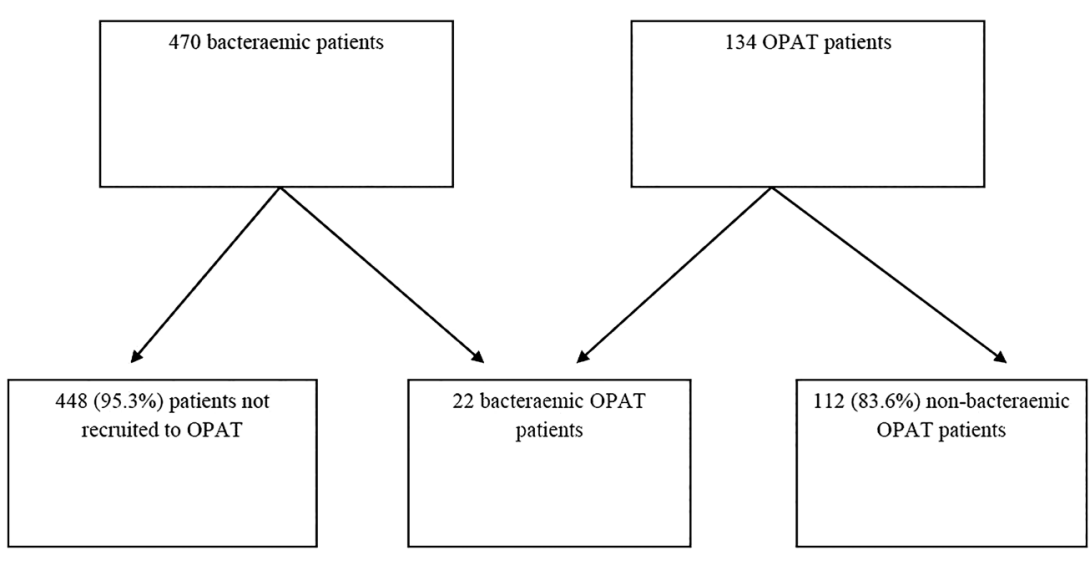


Table 1 Demographic and clinical data on 470 patients, with 556 associated bacteraemic or fungaemic episodes, who did and did not receive OPAT

\begin{tabular}{|c|c|c|c|}
\hline & \multicolumn{2}{|l|}{ OPAT } & \multirow[b]{2}{*}{ p Value* } \\
\hline & Yes & No & \\
\hline Patients & 22 & 448 & \\
\hline Infections (Bacteraemic/fungaemic episodes) & 25 & 531 & \\
\hline \multicolumn{4}{|l|}{ Aget (years), n (\%) } \\
\hline $16-30$ & $2(9.1)$ & $38(8.5)$ & 0.621 \\
\hline $31-50$ & $7(31.8)$ & $99(22.1)$ & \\
\hline $51-70$ & $8(36.4)$ & $156(34.8)$ & \\
\hline$>70$ & $5(22.7)$ & $155(34.6)$ & \\
\hline \multicolumn{4}{|l|}{ Gendert, n (\%) } \\
\hline Male & $11(50.0)$ & $262(58.5)$ & 0.431 \\
\hline Female & $11(50.0)$ & $186(41.5)$ & \\
\hline \multicolumn{4}{|l|}{ Place of acquisition, $\mathrm{n}(\%)$} \\
\hline CA & $20(90.9)$ & $188(35.4)$ & $<0.001$ \\
\hline HCA & $2(9.1)$ & $198(37.3)$ & \\
\hline HA & 0 & $143(26.9)$ & \\
\hline Not defined & 0 & $2(0.4)$ & \\
\hline \multicolumn{4}{|l|}{ Specialty, n (\%) } \\
\hline Medicine & $22(88.0)$ & $430(81.0)$ & $<0.001$ \\
\hline Surgery & $2(8.0)$ & $101(19.0)$ & \\
\hline O\&G & $1(4.0)$ & $0(0.0)$ & \\
\hline \multicolumn{4}{|l|}{ Sites of infection, $\mathrm{n}(\%)$} \\
\hline CVC (uncomplicated) & & & 0.129 \\
\hline Tunnelled & 0 & $33(6.2)$ & \\
\hline Non-tunelled & $2(8.7)$ & $27(5.1)$ & \\
\hline CVC $\neq$ (complicated/metastatic spread) & $2(8.0)$ & $21(4.1)$ & \\
\hline Peripheral cannula & & $1(0.2)$ & \\
\hline Urinary tract (catheter-associated) & & $58(10.9)$ & \\
\hline Urinary tract§ (non-catheter-associated) & $11(44.0)$ & $146(27.5)$ & \\
\hline Biliary tract & $3(12.0)$ & $51(9.6)$ & \\
\hline GIๆ tract & $3(12.0)$ & $32(6.0)$ & \\
\hline GU tract & & $9(1.7)$ & \\
\hline Liver abscess & & $9(1.7)$ & \\
\hline LRT (non-ventilator-associated) & & $35(7.0)$ & \\
\hline LRT (ventilator-associated) & & $4(0.8)$ & \\
\hline Skin and soft tissue infection & $3(12.0)$ & $26(4.9)$ & \\
\hline Peripheral joints (native) & $1(4.0)$ & $2(0.4)$ & \\
\hline Peripheral joints (prosthetic) & & $1(0.2)$ & \\
\hline Meningitis & & $3(0.6)$ & \\
\hline Not defined & & $73(13.8)$ & \\
\hline \multicolumn{4}{|l|}{ Mortality, n (\%) } \\
\hline Inpatient & 0 & $22(4.9)$ & 0.287 \\
\hline 7-day & 0 & $12(2.7)$ & 0.437 \\
\hline 30-day & 0 & $24(5.4)$ & 0.265 \\
\hline
\end{tabular}

One patient had a bacteraemic and non-bacteraemic episode, so this was recorded in both groups.

${ }^{*} \chi^{2}$ test.

tPatient-specific variables. Age and gender were reported as a percentage of patients.

‡Complicated CVC or metastatic infections occurred only in renal haemodialysis patients. This included vertebral column, infective endocarditis and pacemaker infection.

$\S$ Two were complicated lower UTIs, one postprostatic biopsy.

ПEnteric fevers $(2 \times S$. paratyphi and $1 \times S$. typhi).

** Metastatic complication of fistula site and catheter-associated UTIs in renal haemodialysis patients.

CA, community-acquired; CVC, central venous catheter; GI, gastrointestinal; GU, genito-urinary; HA, hospital-acquired; HCA, health-care associated; LRT, lower respiratory tract; 0\&G,

obstetrics and gynaecology; OPAT, outpatient parenteral antibiotic treatment; UTIs, urinary tract infections.

(SSTI), $3 / 29(10.3 \% ; 95 \%$ CI $2.2 \%$ to $27.4 \%)$, gastrointestinal tract infection (all enteric fevers), $3 / 8(37.5 \% ; 95 \%$ CI $8.5 \%$ to $75.5 \%)$ and biliary tract infection, $3 / 54$ (5.6\%; CI $95 \% 1.2 \%$ to $15.4 \%)$. There was no statistically significant difference in 30-day mortality between bacteraemic patients receiving OPAT and those not receiving OPAT; 0 vs $24 / 470(5.4 \%$; $95 \%$ CI $3.3 \%$ to $7.5 \%$ ).

\section{OPAT patients}

Of the 134 patients who received OPAT, $22(16.4 \%$; 95\% CI $10.5 \%$ to $23.6 \%$ ) were bacteraemic. All these patients were recruited through the blood culture database and were not referred directly from clinical teams. There was one patient with a bacteraemia and a non-bacteraemic episode so this information was recorded in both groups. 
Demographic and clinical data of all OPAT patients are summarised in table 2. UTI, $11 / 51 \quad(21.6 \%$; 95\% CI $11.3 \%$ to $35.3 \%$ ) was the most common cause of bacteraemia in OPAT patients. Unadjusted data demonstrated significant differences in sites of infection, with more upper UTIs in the bacteraemic group compared with the non-bacteraemic group, 9/22 (40.9\%) vs $26 / 123(21.1 \%), p=0.046$. There were no deaths in either bacteraemic or non-bacteraemic patients and no significant difference in relapse rates at 30 days; $1 / 22$ (4.6\%) vs $5 / 112$ $(4.5 \%)$

Table 2 Demographic and clinical data on 134 patients who received OPAT (145 treatment episodes), with bacteraemia and without bacteraemia

\begin{tabular}{|c|c|c|c|}
\hline & $\begin{array}{l}\text { Without } \\
\text { bacteraemia }\end{array}$ & $\begin{array}{l}\text { With } \\
\text { bacteraemia }\end{array}$ & p Value* \\
\hline Patients & 112 & 22 & \\
\hline Episodes & 123 & 22 & \\
\hline Aget (years), n (\%) & & & 0.777 \\
\hline $16-30$ & 20 (17.9) & $2(9.1)$ & \\
\hline $31-50$ & $35(31.3)$ & $7(31.8)$ & \\
\hline $51-70$ & $35(31.3)$ & $8(36.4)$ & \\
\hline$>70$ & $22(19.6)$ & $5(22.7)$ & \\
\hline Gendert, n (\%) & & & 0.701 \\
\hline Male & $51(45.5)$ & $12(50.0)$ & \\
\hline Female & $61(54.5)$ & $11(50.0)$ & \\
\hline Specialty, n (\%) & & & 0.941 \\
\hline Medical & $109(88.6)$ & $19(86.4)$ & \\
\hline Surgical & $10(8.1)$ & $2(9.1)$ & \\
\hline O\&G & $4(3.3)$ & $1(4.6)$ & \\
\hline Site of infection, $\mathrm{n}(\%)$ & & & 0.025 \\
\hline Urinary tract (upper) & $26(21.1)$ & $9(40.9)$ & \\
\hline Urinary tract (lower) & 14 (11.4) & $2 \ddagger(9.1)$ & \\
\hline Biliary tract & 0 & $2(9.1)$ & \\
\hline Skin and soft tissue & $57(46.3)$ & $2(9.1)$ & \\
\hline Central venous catheter & 0 & $2(9.1)$ & \\
\hline Gl tract & $2(1.6)$ & $3(13.6)$ & \\
\hline Infective endocarditis & 0 & $1(4.6)$ & \\
\hline LRT & $1(0.8)$ & 0 & \\
\hline Meninges & $2(1.6)$ & 0 & \\
\hline Orthopaedic infections & $6(4.9)$ & $1(4.6)$ & \\
\hline Vertebral column (VC) & 2 & 0 & \\
\hline Osteomyelitis (non-VC) & 0 & 0 & \\
\hline Peripheral joints & 3 & 1 & \\
\hline Sternal wound & 1 & 0 & \\
\hline Other & $15 \S(12.2)$ & 0 & \\
\hline Outcomet, n (\%) & & & 0.987 \\
\hline Recovered/anticipated outcome & $107(95.5)$ & $21(95.5)$ & \\
\hline Relapse & $5(4.5)$ & $1(4.6)$ & \\
\hline $\begin{array}{l}\text { Death within } 30 \text { days of } \\
\text { treatment completion, } \mathrm{N}(\%)\end{array}$ & 0 & 0 & $\mathrm{~N} / \mathrm{a}$ \\
\hline \multicolumn{3}{|c|}{ Duration of intravenous treatment (days) } & 0.207 \\
\hline Mean (SD) & $8.6(9.5)$ & $6.2(4.0)$ & \\
\hline Median (max-min) & $7.0(0.0-64.0)$ & $6.0(0.0-14.0)$ & \\
\hline
\end{tabular}

${ }^{*} \chi^{2}$ test for categorical variables, t-test for continuous variables.

†Patient-specific variables so reported as a percentage of patients. One patient is in both columns because they had one episode without bacteraemia and one with bacteraemia.

¥One postprostatic biopsy.

§Other included liver abscesses, malignant otitis externa, meningitis, leptospirosis, infected ovarian cysts, prepatella bursitis, fistula infection, tuberculosis, bronchiectasis and community-acquired pneumonia with empyema.

$\mathrm{GI}$, gastrointestinal; LRT, lower respiratory tract; $0 \& \mathrm{G}$, obstetrics and gynaecology; OPAT, outpatient parenteral antibiotic treatment.
Total number of days on intravenous antibiotics received out of hospital was 1198, roughly equivalent to the number of bed days saved. The most common drugs administered were either intravenous ceftriaxone once daily or ertapenem. In bacteraemic and non-bacteraemic groups, median (IQR) duration of intravenous treatment was non-significantly different, 7 (5-8) vs 6 (3-8).

\section{Microbiology}

For all bacteraemic patients, 377 (67.8\%) blood culture isolates were Gram-negative. The most common organisms were E. coli and Staphylococcus aureus (table 3). More patients with E. coli bacteraemia were recruited to OPAT compared with other blood culture isolates and, among these, ESBL production was significantly higher in the OPAT group compared with the non-OPAT group, 9/11(81.8\%) vs $17 / 192(8.9 \%), \mathrm{p}<0.001$.

\section{Bacteraemic patient characteristics and recruitment into OPAT}

Univariate and multivariate logistic regression analysis to investigate the association between bacteraemic patient characteristics and those recruited to OPAT is reported in table 4. There were no statistically significant associations in univariate or multivariate logistic regression analysis. In a subgroup analysis of patients with Gram-negative bacteraemia, ESBL production was strongly associated with OPAT recruitment, OR 5.85; 95\% CI 1.94 to 17.58, $\mathrm{p}=0.002$ (table 5).

\section{DISCUSSION}

This is the first paper to examine the utility of a blood culture database to identify patients suitable for OPAT. An additional $16.4 \%$, or 22 extra patients, were recruited to OPAT through the blood culture database. Without this 'prompt', clinical assessment of bacteraemic patients for OPAT suitability is unlikely to have occurred. Although a useful adjuvant to a clinical referral system, more patients were probably not recruited due to severity of infection, as bacteraemic patients often

Table 3 Bacteraemia isolates on 470 patients, with 556 associated bacteraemic or fungaemic episodes, receiving or not receiving OPAT

\begin{tabular}{|c|c|c|c|}
\hline & \multicolumn{2}{|l|}{ OPAT } & \multirow[b]{2}{*}{ p Value* } \\
\hline & Yes $(\mathrm{N}=25)$ & No $(\mathrm{N}=531)$ & \\
\hline Escherichia coli, n (\%) & $11(44.0)$ & $192(36.2)$ & 0.426 \\
\hline $\mathrm{ESBL}+\mathrm{ve}$ & 9 & 19 & $<0.001 \dagger$ \\
\hline ESBL -ve & 2 & 173 & \\
\hline K. pneumonia, n (\%) & $2(8.0)$ & $51(9.7)$ & 0.812 \\
\hline $\mathrm{ESBL}+\mathrm{ve}$ & 0 & 14 & $0.388 \dagger$ \\
\hline ESBL -ve & 2 & 37 & \\
\hline$P$. aeruginosa, $\mathrm{n}(\%)$ & 0 & $24(4.5)$ & 0.277 \\
\hline MSSA, n (\%) & $2(8.0)$ & $51(9.7)$ & 0.789 \\
\hline MRSA, n (\%) & 0 & $3(0.6)$ & 0.706 \\
\hline Candida species, n (\%) & $1(4.0)$ & $9(1.7)$ & 0.397 \\
\hline Otherf, n (\%) & $9(36.0)$ & $202(38.0)$ & 0.837 \\
\hline \multicolumn{4}{|c|}{$\begin{array}{l}\text { One patient had a bacteraemic and non-bacteraemic episode, so this was recorded in } \\
\text { both groups. } \\
\text { *Pearsons } \chi^{2} \text { test. Tests each bacteraemic isolate verses all other bacteraemic isolate. } \\
\text { tESBL +ve versus ESBL -ve for each bacteraemic isolate. } \\
\text { †These include two S. paratyphi, one } S \text {. typhi, one } P \text {. mirabilis, one } E \text {. aerogenes, one } \\
\text { Group G streptococcus, one Streptococcus viridans, one Acinetobacter and one } \\
\text { Candida haemulonii. } \\
\text { ESBL, extended-spectrum } \beta \text {-lactamases; MRSA, methicillin-resistant Staphylococcus } \\
\text { aureus; MSSA, methicillin-susceptible S. aureus; OPAT, outpatient parenteral antibiotic } \\
\text { treatment. }\end{array}$} \\
\hline
\end{tabular}


Table 4 Univariate and multivariate logistic regression analyses for all bacteraemic patients to investigate association between patient characteristics and recruitment to OPAT (469 patients, 555 bacteraemic patients)

\begin{tabular}{|c|c|c|c|c|}
\hline & \multicolumn{2}{|l|}{ Univariate analysis } & \multicolumn{2}{|c|}{ Multivariate analysis } \\
\hline & OR $(95 \% \mathrm{Cl})$ & p Value* & OR (95\% Cl) & p Value* \\
\hline \multicolumn{5}{|l|}{ Age (years) } \\
\hline $16-30$ & Reference & 0.733 & Reference & 0.633 \\
\hline $31-50$ & $1.18(0.23$ to 6.06$)$ & & $1.12(0.22$ to 5.80$)$ & \\
\hline $51-70$ & $1.02(0.21$ to 4.98$)$ & & 0.91 (0.18 to 4.45$)$ & \\
\hline$>70$ & $0.61(0.12$ to 3.28$)$ & & 0.51 (0.09 to 2.79) & \\
\hline \multicolumn{5}{|l|}{ Gender } \\
\hline Female & Reference & 0.593 & Reference & 0.799 \\
\hline Male & 0.79 (0.34 to 1.86$)$ & & 0.89 (0.37 to 2.12$)$ & \\
\hline \multicolumn{5}{|l|}{ Specialty } \\
\hline Medicine & Reference & 0.254 & Reference & 0.237 \\
\hline Surgery & $0.43(0.10$ to 1.83$)$ & & $0.42(0.10$ to 1.78$)$ & \\
\hline \multicolumn{5}{|l|}{ Site of infection } \\
\hline Urinary tract & Reference & 0.415 & Reference & 0.349 \\
\hline Skin and soft tissue & 1.64 (0.40 to 6.77$)$ & & 1.54 (0.36 to 6.67$)$ & \\
\hline Other & $0.67(0.27$ to 1.62$)$ & & $0.61(0.24$ to 1.52$)$ & \\
\hline
\end{tabular}

Table 5 Univariate and multivariate logistic regression analyses for Gram-negative bacteraemic patients to investigate the association between patient characteristics, blood culture isolates and recruitment to OPAT (332 patients, 377 bacteraemic patients)

\begin{tabular}{|c|c|c|c|c|}
\hline & \multicolumn{2}{|l|}{ Univariate analysis } & \multicolumn{2}{|l|}{ Multivariate analysis } \\
\hline & OR $(95 \% \mathrm{Cl})$ & p Value* & OR (95\% Cl) & p Value* \\
\hline \multicolumn{5}{|l|}{ Age (years) } \\
\hline $16-30$ & Reference & 0.464 & Reference & 0.547 \\
\hline $31-50$ & $1.56(0.17$ to 14.11$)$ & & $1.67(0.17$ to 16.42$)$ & \\
\hline $51-70$ & $1.03(0.12$ to 8.94$)$ & & $0.97(0.10$ to 9.12$)$ & \\
\hline$>70$ & $0.47(0.05$ to 4.77$)$ & & $0.53(0.05$ to 5.76$)$ & \\
\hline \multicolumn{5}{|l|}{ Gender } \\
\hline Female & Reference & 0.442 & Reference & 0.614 \\
\hline Male & $0.67(0.24$ to 1.88$)$ & & $0.76(0.25$ to 2.25$)$ & \\
\hline \multicolumn{5}{|l|}{ Specialty } \\
\hline Medicine & Reference & 0.242 & Reference & 0.228 \\
\hline Surgery & 0.30 (0.04 to 2.28$)$ & & 0.28 (0.03 to 2.23$)$ & \\
\hline \multicolumn{5}{|l|}{ Site of infection } \\
\hline Urinary tract & Reference & 0.341 & Reference & \\
\hline Skin and soft tissue & $4.69(0.49$ to 44.82$)$ & & $5.94(0.39$ to 89.49$)$ & \\
\hline Other & $0.85(0.29$ to 2.49$)$ & & $0.73(0.22$ to 2.41$)$ & 0.344 \\
\hline \multicolumn{5}{|l|}{ Escherichia coli } \\
\hline No & Reference & 0.987 & Reference & 0.756 \\
\hline Yes & 1.01 (0.36 to 2.83$)$ & & 1.21 (0.37 to 3.95$)$ & \\
\hline \multicolumn{5}{|l|}{ ESBL production } \\
\hline Negative & Reference & 0.001 & Reference & 0.002 \\
\hline Positive & $6.15(2.13$ to 17.75$)$ & & 5.85 (1.94 to 17.62$)$ & \\
\hline
\end{tabular}

require resuscitation in hospital. Also, many patients admitted from the community can be switched to oral alternatives and, in the absence of drug resistance, do not require prolonged intravenous therapy. Our data show that patients with community-onset and multidrug resistant (MDR) infections were most likely to be recruited to OPAT, and none of these patients were directly referred without prompting through the clinical referral system.

The most common sites of infection in patients recruited to OPAT were SSTIs and UTIs. A 2-year retrospective review of 
patients treated with OPAT in one Scottish centre found the majority of infections were SSTIs, 125 (59\%) of 212 episodes. $^{5}$ All were identified clinically and, in our study, we also found that these patients were predominantly recruited by clinical assessment rather than blood culture findings.

In contrast, the blood culture database was particularly useful in identifying patients with MDR UTIs, a condition that can't be diagnosed clinically, and with these infections a positive blood culture result triggered a clinical assessment which wouldn't have otherwise occurred. One paper retrospectively reviewed the use of OPAT to facilitate early discharge of patients with UTI caused by ESBL producing Enterobacteriaceae. In this small retrospective study, 11 patients with 25 treatment episodes, it is unclear whether any of these bacteraemic patients were identified and recruited following the validation of the blood culture result. The authors concluded that OPAT administration of ertapenem was effective and decreased costs associated with MDR UTIs, ${ }^{15}$ a finding similar to ours.

The most common bacteraemic isolate in our OPAT cohort was E. coli. Enhanced surveillance of E. coli bacteraemia has been mandatory for NHS acute trusts since June 2011. ${ }^{16}$ In England, the incidence of bacteraemia is 56/100 000 and $12 \%$ are ESBL producers or MDR. In 2015, 37275 bacteraemic episodes were reported and approximately 3000 bacteraemic episodes were either ESBL producers or required intravenous antibiotics due to resistance to all suitable oral agents. If, based on our data, a third of bacteraemic episodes could be treated out of hospital ( 1000/annum) for an average of 5 days, then a possible 5000 hospital bed days could be saved in England each year.

Our blood culture database was also useful in identifying other medical conditions not normally treated with intravenous antibiotics out of hospital, including enteric fevers, biliary tract infections and central venous catheter-associated infections where line salvage was being attempted. In the absence of a blood culture result it is unlikely these patients would have been recruited to OPAT.

There were no deaths in the OPAT group with bacteraemia, despite bacteraemia being a marker of severe infection. Many of these patients were recruited after hospital admission and then stabilised before discharge on an intravenous antibiotic. In addition to reducing length of inpatient stay, our data demonstrate the safety of this approach. Among bacteraemic patients recruited to OPAT, all had community-onset infections. Hospital-acquired infections are generally medical device related or procedure related, and more commonly occur in patients in critical care areas, ${ }^{17}$ so it is not surprising that the majority of patients recruited had community-acquired bacteraemia.

There were limitations to this study. Before and during this study, we did not record information on the proportion of unsuitable patients referred to OPAT, nor did we have prestudy data on our OPAT mix including bacteraemic patients. Because of our proactive approach, it is possible patients were identified before clinical teams had time to refer (which, in part, is dependent upon awareness of OPAT services within our NHS trust). The numbers of bacteraemic patients recruited to OPAT were small and as there were no deaths in the 'bacteraemic' and 'non-bacteraemic' groups, a survival analysis was not possible. In logistic regression analysis for bacteraemic patients, we were unable to include place of acquisition in the model as no patients with hospital-acquired infection were recruited. In a larger study, where there were patients with hospital-acquired infection, we could have tested the association between community-onset bacteraemia and OPAT recruitment. The sample size was, however, big enough to demonstrate significant differences in unadjusted and adjusted data.

In summary, our study demonstrates that a blood culture database provided a useful adjuvant to a clinical referral system for OPAT recruitment. The blood culture database was particularly useful for prompting the identification of patients with community-onset infections and MDR UTIs caused by ESBL-producing E. coli. Bacteraemic patients received treatment safely and all had good clinical outcomes. Within the UK, each year, clinical assessment of all patients with ESBL-producing E. coli bacteraemia could identify hundreds of hospitalised patients suitable for OPAT recruitment, with the potential to reduce length of inpatient stay without compromising patient safety and clinical outcomes.

\section{Main messages}

As a blood culture database provides a useful adjuvant to a clinical referral system, an infection specialist (including a microbiologist) can prompt or make outpatient parenteral antibiotic treatment (OPAT) referrals.

- This is especially useful for community-onset, extended-spectrum $\beta$-lactamase (ESBL)-producing or multidrug resistant Escherichia coli urinary tract infections (UTIs) that cause bacteraemia.

- All bacteraemic patients recruited to OPAT received safe treatment and had good clinical outcomes.

\section{Current research questions}

- Would a standardised comment, appended to extended-spectrum $\beta$-lactamase (ESBL)-producing Escherichia coli blood culture reports, increase outpatient parenteral antibiotic treatment (OPAT) recruitment and safely decrease length of inpatient stay?

- What would be the national effect on length of inpatient stay if a standardised comment was automatically attached to ESBL-producing $E$. coli blood culture results in all UK NHS trusts?

- Other than blood cultures, how useful are significant microbiology results in prompting assessment for OPAT recruitment?

Acknowledgements The authors thank the specialist registrars at the Royal London Hospital, fast response team and Tower Hamlet community nurses who helped to collect this data.

Contributors MM collected and analysed data in conjunction with LMP. Statistical analysis was performed by CW.

Competing interests None declared.

Ethics approval The study was approved by the clinical governance committee at Barts Health NHS Trust.

Provenance and peer review Not commissioned; externally peer reviewed.

\section{REFERENCES}

1 Barr DA, Semple L, Seaton RA. Outpatient parenteral antimicrobial therapy (OPAT) in a teaching hospital-based practice: a retrospective cohort study describing experience and evolution over 10 years. Int J Antimicrob Agents 2012;39:407-13 
2 Chapman ALN, Dixon S, Andrews D, et al. Clinical efficacy and cost-effectiveness of outpatient parenteral antibiotic therapy (OPAT): a UK perspective. J Antimicrob Chemother 2009;64:1316-24.

3 Gray A, Dryden $M$, Charos A. Antibiotic management and early discharge from hospital: An economic analysis. J Antimicrob Chemother 2012;67:2297-30.

4 Laupland KB, Valiquette L. Outpatient parenteral antimicrobial therapy. Can J Infect Dis Med Microbiol 2013;24:9-11.

5 Nathwani D. The management of skin and soft tissue infections: outpatient parenteral antibiotic therapy in the United Kingdom. Chemotherapy 2001;47(Suppl 1):17-23.

6 Chapman ALN, Seaton RA, Cooper MA, et al. Good practice recommendations for outpatient parenteral antimicrobial therapy (OPAT) in adults in the UK: a consensus statement. J Antimicrob Chemother 2012;67:1053-62.

7 Tice $A D$, Rehm SJ, Dalovisio JR, et al. Practice guidelines for outpatient parenteral antimicrobial therapy. Clin Infect Dis 2004;38:1651-71.

8 Five year forward view (NHS England, October 2014). https://www.england.nhs.uk/ wp-content/uploads/2014/10/5yfv-web.pdf (accessed 26 Jan 2016).

9 Esposito S, Noviello S, Leone $S$, et al. Outpatient parenteral antibiotic therapy (OPAT) in different countries: a comparison. Int J Antimicrob Agents 2004;24:473-8.

10 Lai $A$, Tran $T$, Nguyen HM, et al. Outpatient parenteral antimicrobial therapy at large Veterans Administration medical center. Am J Manag Care 2013;19:e317-24.
11 Allison GM, Muldoon EG, Kent DM, et al. Prediction model for 30-day hospital readmissions among patients discharged receiving outpatient parenteral antibiotic therapy. Clin Infect Dis 2014;58:812-19.

12 Andrews MM, von Reyn CF. Patient selection criteria and management guidelines for outpatient parenteral antibiotic therapy for native valve infective endocarditis. Clin Infect Dis 2001;33:203-9.

13 Laupland KB. Incidence of bloodstream infection: a review of population-based studies. Clin Microbiol Infect 2013;19:492-500.

14 Garner JS, Jarvis WR, Emori TG, et al. CDC definitions for nosocomial infections, 1988. Am J Infect Control 1988;16:128-40.

15 Bazaz R, Chapman AL, Winstanley TG. Ertapenem administered as outpatient parenteral antibiotic therapy for urinary tract infections caused by extended-spectrum-beta-lactamase-producing Gram-negative organisms. J Antimicrob Chemother 2010;65:1510-13.

16 Public Health England. Escherichia coli (E. coli) bacteraemia: annual dataPublications.... https://www.gov.uk/government/statistics/escherichia-coli-e-colibacteraemia-annual-data (accessed 9 Aug 2016).

17 Melzer M, Wickramasinghe D, Welch C. Outcomes in consecutive hospitalised UK patients with bacteraemia or fungaemia caused by medical devices and procedures. J Hosp Infection 2015;91:146-52. 